\title{
Enseñar y aprender en la no presencialidad: percepciones de estudiantes de posgrado-docentes
}

\author{
Cariaga, Romina
}

\section{Resumen}

Este trabajo tiene por objetivo hacer un análisis contextualizado de los relatos de experiencia manifiestos en un foro sobre las prácticas educativas en modalidad no presencial durante la pandemia de docentes-estudiantes de posgrado. Se apoya en herramientas de la investigación cualitativa interpretativa y propone un análisis de los relatos desde dos dimensiones: dificultades encontradas y cambios implementados. Los resultados muestran que si bien los recursos tecnológicos adoptados en este contexto excepcional no reemplazan la potencialidad de los vínculos ni los métodos de la presencialidad se constituyen en recursos necesarios y enriquecedores de todas las propuestas áulicas. Asimismo, se concluye que es necesario pensar en nuevas formas de escolaridad, híbridas, donde lo virtual y lo presencial coexistan y donde las instituciones procuren la formación de sujetos con capacidades para desenvolverse en entornos cambiantes, dispuestos a aprender de otros y con otros a través de una adopción crítica de la tecnológica.

Palabras clave: no presencialidad; recursos TIC; dificultades; cambios

Este trabajo está enmarcado en el proyecto de investigación «El aula virtual y sus actores en la educación universitaria» PI N 04/V113, del CURZA-UNCo. (Argentina). Recibido el 01/12/2020, aceptado el 05/05/2021 y publicado el 07/07/2021.

DOI: https://doi.org/10.33255/3262/933

Autoría: *Centro Universitario Regional Zona Atlántica, Universidad Nacional del Comahue (Argentina). Contacto: rominacariaga@gmail.com 


\title{
Teaching and learning in non-face-to-face modality: perceptions of postgraduate students-teachers
}

\begin{abstract}
This work aims to make a contextualized analysis of experience reports posted in a forum by postgraduate students, who are also teachers, about their educational practices in non-face-to-face modality during the pandemic. Qualitative interpretive research tools support this study and propose an analysis of the narrations from two dimensions: encountered difficulties and implemented changes. The results show that although the technological resources adopted in this exceptional scenario do not replace the potential of the links or the methods of face-to-face teaching and learning, they constitute necessary and enriching resources for any kind of educational proposal. Likewise, this paper concludes that thinking about new forms of hybrid schooling, where virtuality and face-to-face modality coexist and where institutions provide students training focused on abilities like working in changing environments, learning from others and with others through a critical adoption of technology is essential.
\end{abstract}

Key words: non-face-to-face modality; technological resources; difficulties; changes

\section{Ensinar e aprender na modalidade não presencial: percepções de estudantes de pós-graduação/professores}

\section{Resumo}

O objetivo deste trabalho é fazer uma análise contextualizada dos relatos de experiência expostos em um fórum sobre as práticas educacionais na modalidade não presencial durante a pandemia, de professores-estudantes de pós-graduação. 0 estudo apoia-se em ferramentas da pesquisa qualitativa interpretativa e propõe uma análise dos relatos a partir de duas dimensões: as dificuldades encontradas e as mudanças implementadas. Os resultados mostram que embora os recursos tecnológicos adotados neste contexto excepcional não substituam a potencialidade dos vínculos nem os métodos da presencialidade, constituem recursos necessários e enriquecedores de todas as propostas educacionais. Assim, conclui-se que é preciso pensar em novas formas de escolarização, híbridas, onde coexistam o virtual e o presencial e onde as instituições procurem formar sujeitos com capacidades para agir em entornos com mudanças, dispostos a aprender de outros e com outros através de uma adoção crítica da tecnologia.

Palavras-chave: ensino não presencial; recursos TIC; dificuldades; mudanças 
Es extraño extrañar lo habitual, lo de todos los días, lo que ocurre casi sin querer, aquello que es, como se dice vulgarmente y encogiéndose de hombros: «lo de siempre», «nada en especial».

CARLOS SKLIAR

\section{Introducción}

Evidentemente, esta nueva escena global provocada por el COVID-19 nos sorprendió por completo. Si bien todos los ámbitos de la vida padecieron los efectos de la pandemia, el campo educativo resultó severamente alterado. El modelo casi universal de escuela se desmoronó sin previo aviso y el cierre de las aulas propició una migración forzada hacia la modalidad no presencial en todos los niveles educativos.

La profundidad de esta situación y sus múltiples efectos hizo claro que era necesario interpelar a diferentes miembros de la comunidad educativa local/ regional para pensar, debatir y contrastar experiencias, percepciones, sentidos y posibles proyecciones futuras. En consecuencia, y dado que el proyecto de investigación del que surge este trabajo focaliza en el estudio de las aulas virtuales del Centro Universitario Regional Zona Atlántica (CURZA) de la Universidad Nacional del Comahue (UNCo), se consideró importante implementar una consulta a través de un foro de discusión a los docentes y al mismo tiempo estudiantes de la carrera de posgrado virtual «Especialización en educación mediada por tecnología digital» (CURZA-UNCo). Las preguntas planteadas como disparadores de la discusión fueron: ¿cómo fue la experiencia de pasar de un día para el otro al modo online? ¿Qué dificultades surgieron? ¿Qué cambios en su práctica debieron implementar? Cuando la pandemia termine, ¿serán ustedes mejores docentes?, ¿peores?, ¿los mismos? Las interrogaciones orientadoras de la reflexión fueron resignificadas en cada contexto.

En esta investigación, entendemos la práctica educativa como una actividad dinámica, reflexiva, que comprende los acontecimientos ocurridos en la interacción entre docente y alumnos y que incluye la intervención pedagógica ocurrida antes y después de esos procesos. Cabe mencionar que si bien los participantes del foro poseían formaciones relativas al uso de las tecnologías digitales en contextos educativos, sus expresiones dieron cuenta de diversas problemáticas a las que debieron hacer frente durante el aislamiento $\mathrm{y} / \mathrm{o}$ distanciamiento social preventivo y obligatorio (AS)DISPO, al mismo tiempo que describieron cambios que debieron realizar en sus prácticas.

Con el fin de interpretar esos enunciados a la luz de lo que sostienen los teóricos, se realizó un relevamiento bibliográfico de las aportaciones de 
distintos autores sobre el tema. Posteriormente, se reconocieron las problemáticas centrales y los cambios más frecuentes en las prácticas sin perder de vista los aspectos teóricos, profundizándolos. Por último, se procedió a sistematizar la información recabada, sintetizarla e interpretarla para arribar a conclusiones globales de acuerdo con los datos disponibles.

\section{Marco teórico}

Para interpretar los relatos de experiencia volcados en el foro de discusión se tuvieron en cuenta aportes de diferentes autores, entendiendo que sus miradas epistemológicas permitirían orientar esta investigación y realizar un examen integrado y comprehensivo de la realidad reflejada en las narraciones docentes. De este modo, pudimos definir las dos dimensiones centrales, con sus respectivas subdimensiones, que guiaron este análisis: por un lado, las dificultades encontradas, y por el otro, los cambios implementados.

Para examinar la dimensión de las dificultades que debieron enfrentar los docentes nos basamos en los diversos «conflictos y tensiones» expuestos por Dussel (2020a) a partir de la «domiciliación» de la escuela, ya no más anclada en sede escolar, sino instalada en lo doméstico y sobre todo en las pantallas. Por otra parte, para entender la naturaleza de los cambios que los docentes dicen haber implementado en sus prácticas educativas en este contexto de no presencialidad, consideramos que los aportes presentados por Scolari (2018) en Las leyes de la interfaz: Diseño, ecología, evolución, complejidad son de gran utilidad y permiten identificar dinámicas, mutaciones y evoluciones. Asimismo, y para ponderar esos cambios en relación con formas y contenidos, creímos necesario incorporar los conceptos vertidos por Area Moreira (2014) sobre las «nuevas alfabetizaciones» y las aportaciones de Cobo (2016) sobre «aprendizajes invisibles». Por último, el entrecruce de estas miradas nos permitió identificar las percepciones docentes respecto de las transformaciones experimentadas e indagar en sus proyecciones futuras.

Dussel es una de las autoras que más claramente aborda el nuevo entorno sociotécnico, la transformación de lo doméstico en espacio de trabajo y el vínculo pedagógico en línea. En numerosos trabajos, la autora describe ampliamente el modo en que la pandemia ha cambiado la «domiciliación» de lo escolar, trasladando la escuela al seno de las dinámicas familiares, transformando a docentes y alumnos en seres domésticos (Dussel, 2020a; Dussel, 2020b; Dussel et al., 2020). Asimismo, plantea que en este nuevo contexto se han generado diversos conflictos y tensiones como los siguientes: la escolarización versus desigualdades sociales, la desaparición física de las aulas versus 
la ausencia de otras voces para aprender juntos, el borramiento de fronteras entre la escuela y la casa, y por último, las plataformas escolares versus la posibilidad de generar y acompañar ciertos procesos de aprendizaje en los alumnos. Según la autora, la primera tensión surge a partir de las enormes desigualdades sociales respecto a la conectividad digital, la comodidad del espacio de trabajo y las posibilidades de las familias de sostener y acompañar los aprendizajes de jóvenes y niños. La segunda tensión tiene que ver con las nuevas condiciones sociotécnicas donde no hay garantizados espacios comunes que permitan interacciones sincrónicas. La tercera tensión está vinculada a la confusión de lo escolar y lo doméstico. Finalmente, la cuarta tensión se plantea ante la dificultad para recuperar lo propio de la vida escolar en las actuales condiciones tecnológicas.

Para analizar los cambios implementados fue necesario recurrir al concepto de «interfaz educativa» propuesto por Scolari $(2018,2019)$. Según el autor, una interfaz es una red de actores humanos, institucionales y tecnológicos que mantiene una serie de relaciones dentro de una serie de procesos. En Las leyes de la interfaz (2018), Scolari propone una exploración de ese universo a partir de diez principios básicos que ayudan a comprender la complejidad de la red sociotécnica. Tomando estas leyes podríamos analizar este escenario complejo de reconversión en el que las interfaces educativas han debido evolucionar y transformarse. La cuarta ley, por ejemplo, postula que las interfaces evolucionan mediante procesos similares a los biológicos. En la quinta ley, Scolari sostiene que las interfaces coevolucionan con sus usuarios. Las interfaces impactan en los usuarios y estos, a su vez, las transforman. En la sexta ley, el autor plantea que las interfaces no se extinguen, sino que se transforman. Las viejas interfaces no se pierden, sino que quedan a disposición para que futuros usuarios las recuperen y las reactiven en nuevos contextos.

Para examinar los cambios que demanda el rediseño de la interfaz educativa en este contexto cultural y tecnológico creemos que los aportes de Area Moreira (2014) resultan centrales. El autor reclama la necesidad del reconocimiento y la incorporación de nuevas alfabetizaciones al sistema educativo. Alfabetizaciones centradas en la adquisición de las competencias de producción y análisis del lenguaje audiovisual, en el dominio del uso de los recursos y lenguajes informáticos, en el desarrollo de habilidades de búsqueda, selección y reconstrucción de la información. Una «multialfabetización» que implique la adquisición de las competencias necesarias tanto para interactuar con la cultura existente como para recrearla de un modo crítico y emancipador.

De igual modo, las contribuciones de Cristóbal Cobo (2016) resultan significativas. El autor señala que el cambio más importante que demanda el actual 
escenario educativo no es tecnológico sino de sentido. Por ese motivo, con el fin de analizar los sentidos y desafíos involucrados en el rediseño de las prácticas educativas durante el (AS)DISPO, asumimos la centralidad de reconocer las formas alternativas de adquirir conocimientos, de recuperar aprendizajes invisibles, generados en contextos diversos, a través de la experiencia, la curiosidad y la observación (Cobo, 2016).

\section{Marco metodológico}

Este trabajo se apoya en herramientas de la investigación cualitativa interpretativa desde una perspectiva etnográfica: el análisis de contenido de fuentes documentales, en este caso relatorías de experiencias desarrolladas en un foro de discusión académica del seminario «Alfabetización digital y nuevas prácticas letradas» en la carrera «Especialización en educación mediada por tecnología digital» (CURZA-UNCo). Adopta una aproximación epistemológica desde los propios actores, estudiantes de una carrera de posgrado y docentes del sistema escolar en los niveles primario, secundario y superior. Pretende explorar sus experiencias de prácticas educativas en modalidad no presencial durante la pandemia.

En la recolección de datos procuramos observar el todo aunque de hecho fuese imposible. Prestamos atención a los detalles y a las señales que proporcionaron los sujetos y que indicaron relaciones significativas. Para el análisis de la muestra tuvimos en cuenta, primero, los relatos y los lenguajes de la experiencia en las narraciones docentes y luego los aportes del marco teórico.

Primeramente analizamos las dificultades que docentes y alumnos mencionaron que debieron atravesar desde los «conflictos y tensiones» presentados por Dussel (2020a), con adaptaciones a nuestro contexto de investigación. Así, consideramos las siguientes dimensiones de análisis con sus respectivas subdimensiones:

1) desigualdades sociales: a) conectividad, b) brecha digital, c) posibilidades de las familias, d) espacio de trabajo

2) desaparición física de las aulas: a) ausencia de otras voces para aprender juntos, b) angustia, c) abandono

3) borramiento de fronteras entre la escuela y la casa: a) dislocación del tiempo y espacio escolar, b) intensificación del trabajo, c) invasión a la intimidad

4) acompañamiento de los procesos de aprendizaje: a) escasa alfabetización digital, b) falta del feedback instantáneo, c) dificultad de atención, d) dificultad para establecer y sostener vínculos 
En segundo lugar, y para interpretar los cambios que los docentes manifiestan haber implementado, utilizamos las siguientes dimensiones y subdimensiones:

1) exploración de las posibilidades de las TIC: a) incorporación de las TIC para la producción del conocimiento, b) desarrollo de nuevas formas de sostener vínculos a través de las TIC, c) recuperación de aprendizajes invisibles 2) reconocimiento de la necesidad de desarrollar nuevas prácticas y competencias: a) redefinición de prácticas alfabetizadoras, b) implementación de nuevas dinámicas de construcción de saberes, c) reestructuración de contenidos

\subsection{Caracterización de los sujetos y la muestra}

La muestra se compone por las participaciones en un foro de discusión de treinta y tres (33) estudiantes de posgrado, todos ellos también docentes que se desempeñan en diferentes niveles educativos. El $52 \%$ de ellos ejerce en el nivel superior: universidades (40\%), institutos de formación docente $(9 \%)$ y en el Instituto Provincial de la Administración Pública (3\%); el $45 \%$, en instituciones de nivel secundario; el $3 \%$, en instituciones de nivel primario. Sus edades oscilan entre los 30 y los 45 años y todos debieron realizar la migración forzosa a la modalidad no presencial durante la (AS)DISPO.

La riqueza de sus relatos de experiencias nos brinda la posibilidad de realizar una aproximación reflexiva tanto de las dificultades y los cambios que debieron implementar en sus prácticas como de sus posibles proyecciones futuras.

\section{Análisis de resultados}

De la lectura de la muestra y su relación con la teoría surgieron las siguientes dimensiones y subdimensiones que permiten el análisis y la posterior interpretación:

\subsection{Dificultades encontradas}

\subsubsection{Desigualdades sociales}

El cierre de las escuelas ha afectado a toda la sociedad, pero de manera más acentuada a la población vulnerable, a la que tiene menos posibilidades de educación al margen de la escuela, que vive en entornos definidos por la pobreza, la baja escolarización y el trabajo informal. Al respecto, un docente expone en el foro: «La cuarentena no hizo más que profundizar las brechas entre aquellos sectores que pueden acceder a una educación de calidad mediada 
por la tecnología y quienes no» (Comunicación personal). Ciertamente, la pandemia ha profundizado los abismos y ha exacerbado las ya muy conocidas desigualdades educativas, y eso se evidencia muy especialmente en las zonas rurales, las periferias pobres de las ciudades, las comunidades aborígenes y los grupos necesitados de una educación especial. Así lo expresa un docente:

La mayoría de los estudiantes de los parajes rurales no tienen acceso a internet desde sus hogares; por otro lado, no cuentan con tecnología apropiada para realizar las tareas [...] En este tiempo hemos podido observar una radiografía social de las desigualdades que no han cambiado a lo largo de la historia en nuestro país... (Comunicación personal)

\subsection{1.a. La conectividad}

Con la interrupción de las clases presenciales, los sectores menos afectados por la crisis continuaron sus actividades en modalidad online, programaron clases, tareas, evaluaciones y consiguieron una relativa contención de su alumnado. En el otro extremo, muchos niños, adolescentes y adultos quedaron solos, perdieron comunicación con sus maestros, excepto cuando estos esforzadamente los siguieron contactando por medios caseros (visitas a los domicilios, llamadas por teléfono o a través de WhatsApp). El $80 \%$ de las participaciones en el foro destacó la desigualdad en el acceso a las tecnologías digitales y a la conectividad de docentes y alumnos como una de las principales dificultades afrontadas en todos los niveles educativos. Una profesora de primaria enuncia en el foro: «En la localidad en la que vivimos muchos no tienen acceso a internet. Trabajar online es en muchos casos imposible tanto para los docentes como para los niños» (Comunicación personal). De igual modo, una docente del nivel terciario manifiesta: «Tenemos muchísimos estudiantes que no tienen acceso a la conectividad y otros que tienen acceso por turnos, porque viven en familias numerosas y deben compartir los pocos recursos tecnológicos que tienen» (Comunicación personal).

\subsection{1.b. La brecha digital}

Esta brecha tiene su correlato en las desigualdades socioeconómicas y de infraestructura de nuestra sociedad. Está conformada por un conjunto de obstáculos que existen para el acceso y el uso igualitario de las TIC. Estos obstáculos no solo implican la falta de disponibilidad de recursos TIC y servicios tecnológicos, sino también la ausencia de habilidades y conocimientos necesarios para el uso adecuado de las TIC. Una participante expresa: 
La mayoría de nuestros estudiantes no cuentan con acceso a internet ni al conocimiento de las herramientas tecnológicas [...] pero muchos docentes tampoco cuentan con ese conocimiento necesario para afrontar las exigencias de trabajar en este nuevo contexto. (Comunicación personal)

Otro comentario sostiene:

También esta brecha digital se produce por diferencias generacionales y los docentes de más edad son los que más la sufren, se sienten lejanos a los usos de las nuevas tecnologías. Muchos sienten que el sistema quiere deprenderse de ellos. (Comunicación personal)

Si bien los docentes que participaron del foro poseían formaciones relativas al uso de las tecnologías digitales en contextos educativos, expusieron que su desafío consistió en movilizar esos conocimientos en función de las tecnologías disponibles en sus hogares y en los hogares de los estudiantes.

\subsection{1.c. Las posibilidades de las familias}

El desigual acceso al trabajo ha marcado grandes diferencias. Para los adultos que continúan asistiendo a sus labores, el cuidado de los menores y el seguimiento de las actividades escolares se transforman en una empresa poco asequible, situación que se agrava si se carecen de redes de apoyo. Para aquellos sectores sociales que sobreviven del campo laboral informal la problemática ha sido más trágica ya que sus ingresos han disminuido drásticamente.

Por otra parte, enseñar es una tarea especializada, no cualquiera logra que alguien comprenda algo complejo o se conecte con un tema enteramente nuevo (Terigi, 2020). Los testimonios recogidos señalan que «muchas de las familias han entrado en desesperación ya que se les hace difícil ayudar y acompañar a sus hijxs en este proceso [...] aparecen reclamos, enojos y todo tipo de sentires» (Comunicación personal). Un docente manifiesta que, para muchos padres, «convertirse en asistentes de los maestros, explicarles a sus hijos lo que no comprenden, ayudarlos a organizar su tiempo, interpretar las consignas, etc., es una tarea imposible» (Comunicación personal).

En cuanto a los jóvenes y adolescentes, los relatos denuncian que muchas familias los han convocado para colaborar con distintas tareas por lo que, extenuados de estas, no consiguen involucrarse en las propuestas educativas. «En general trabajan en las asignaturas que les resultan más interesantes, están cansados de las tareas que desarrollan y muchos usan las TIC con otros fines» (Comunicación personal). Al mismo tiempo, una docente del nivel superior indica: 
Hemos visto que muchísimos estudiantes se han dado de baja, por inconvenientes económicos, por contar con un sola computadora que ahora debía ser compartida con varios miembros de la familia, por no llegar con tiempos en cuanto a planificación, correcciones en su trabajo, etc., y lectura y entrega de actividades que exigía cada cátedra, etc. (Comunicación personal)

\subsection{1.d. Espacio de trabajo}

El estudiar y/o trabajar «desde casa» partió del supuesto de que el hogar era un lugar acondicionado para hacerlo. Sin embargo, los testimonios hacen visible que en muchos casos esto no es así. «Hemos podido observar la precariedad de las viviendas de muchos de nuestros alumnos, los escasos recursos económicos con los que cuentan; es prácticamente imposible estudiar en ese contexto» (Comunicación personal).

Por otra parte, el aislamiento implicó para los adultos asumir tareas domésticas, atención infantil, atención de las personas mayores (que se convirtieron en grupo de riesgo) y también las responsabilidades extra en la ayuda con las tareas escolares de los niños del hogar. Los enunciados en el foro reflejan que tanto enseñar como aprender desde casa no ha resultado fácil para nadie. «Descubrí que es muy difícil estudiar y hacer mi trabajo con facilidad desde la mesa del comedor. Siempre hay distracciones en casa» (Comunicación personal).

La pandemia, con sus secuelas de emergencia sanitaria, aislamiento y virtualización de lo cotidiano, quitó el velo a las desigualdades y brechas preexistentes vinculadas a la estructuración social, la pobreza y la fragmentación, y las recrudeció. Al mismo tiempo, generó nuevas distancias sociales, relacionadas con la disponibilidad, acceso y uso de la tecnología. En su conjunto, los docentes describen en sus relatos que el sistema educativo local recibió la pandemia debilitado y retrasado en la realización de cambios que requería de manera inminente. Al respecto, un docente manifiesta: «La educación actual está desfasada y fuera de tiempo, esto lo digo como docente y como el estudiante que soy» (Comunicación personal).

Estas expresiones nos señalan que en el camino hacia la superación de las brechas mencionadas, el Estado tiene un papel fundamental a través del diseño e implementación de políticas económicas, culturales y educativas que apunten a amortiguar esas desigualdades estructurales que hoy afectan más que nunca a docentes, estudiantes y familias.

\subsubsection{Desaparición física de las aulas}

La escuela ha sido históricamente un espacio de encuentro con otros, un territorio de posibilidades, el lugar para interpretar, comprender, experimentar y 
producir juntos. Desde sus orígenes, ha configurado un contexto específico de aprendizaje que produce formas también específicas de aprendizaje, el aprendizaje escolar. En la escuela se aprenden ciertos contenidos, de cierto modo, en un cierto ritmo, y de un cierto modo también se muestra lo que se ha aprendido. Pero hoy la escuela funciona en un contexto diferente; ha migrado a un nuevo territorio en tiempo record y sin posibilidades de una paulatina adaptación al nuevo entorno. En la muestra se observan referencias a las siguientes consecuencias acarreadas por el pasaje compulsivo:

\subsection{2.a. Ausencia de otras voces para aprender juntos}

Con la desaparición de la escuela como espacio colectivo de aprendizajes no hay garantizados espacios comunes que permitan interacciones sincrónicas. El alumno que no posee las condiciones sociotécnicas necesarias no asiste a la escuela, se desconecta del discurso áulico y se aleja del aprendizaje grupal. Al respecto, una docente expone: «Intentamos con clases por Zoom, pero las propuestas sincrónicas son un problema cuando hay falta de conectividad o cuando los/as estudiantes no disponen de computadora» (Comunicación personal). Asimismo, son varios los relatos que describen la «extrañeza» de la nueva escena pedagógica virtualizada: «Los/as estudiantes quieren ver a sus compañeros y al docente, escucharlos, incluso cuando no se animen a hablar o mostrarse en el video para no entrar en la intimidad de sus hogares. Extrañan la antigua vida escolar» (Comunicación personal).

\subsection{2.b. Angustia}

La sensación que tienen estudiantes y docentes es que han perdido la escuela, perdieron las aulas, el espacio de encuentro, de intercambio y de socialización. La palabra «angustia» aparece en diez relatos en el foro: «Nos falta el cara a cara y eso me angustia» (Comunicación personal); «Me angustia ver la desigualdad, las realidades familiares, económicas y tecnológicas de mis alumnos» (Comunicación personal); «A nivel personal me tiene un poco triste, me angustia esta sensación de trabajar en soledad, y puedo advertir que a los alumnos les pasa lo mismo» (Comunicación personal).

En este contexto de incertidumbre, se extrañan las rutinas escolares, sus rituales de ingreso a clase, escuchar al docente, hacer las tareas, etc. Más allá del uso de pantallas replicadoras de la escena escolar, de chats que emulan las discusiones de clase, al parecer nada suple el aula presencial donde sucede el trabajo pedagógico. 


\subsection{2.c. Abandono}

En este escenario, muchos alumnos tienen grandes dificultades para comunicarse con la escuela y han suspendido repentinamente los vínculos pedagógicos que los unían a ella, por lo que resulta necesario trabajar con la hipótesis de un aumento considerable de abandono escolar. Los docentes manifiestan en el foro que en promedio un $50 \%$ de sus alumnos de todos los niveles se ha desconectado de la escuela y es probable que al menos la mitad de ellos ya no regrese, o que no lo haga de inmediato. Diferentes voces revelan con tristeza: «Soy docente en IFDC [...] traté de acercarme a los estudiantes, de alentarlos en sus cursadas, pero muchos abandonaron avisando que volverían cuando mejorara la situación» (Comunicación personal). «Soy docente de nivel medio y universitario. Las respuestas son mínimas, solo contamos con un $60 \%$ de la matrícula activa» (Comunicación personal).

La ausencia de espacios comunes que permitan interacciones sincrónicas favorece una mayor individualización del trabajo pedagógico. La escuela dejó de ser el espacio colectivo de aprendizajes para todos los chicos y las nuevas condiciones sociotécnicas colaboran en la desvinculación de los alumnos de sectores más pobres. Como describe Carlos Skliar (2020), se extraña la escuela:

Se extraña lo común porque es extraña la sensación de no habitar un lugar y un tiempo juntos para hacer las cosas que solo podemos hacer en las escuelas... Esas cosas que no están en ningún otro lugar ni en ningún otro tiempo, que tienen la potencia de la conversación entre varios, que se descubren porque son enseñadas y no solamente encontradas por el azar de una búsqueda azarosa.

\subsubsection{Borramiento de fronteras entre la escuela y la casa}

Llevó siglos de lucha diferenciar instituciones como la familia, el trabajo y la educación, y hoy, gracias a las medidas sanitarias contra la pandemia, se ven superpuestas y en ocasiones confundidas. Al cerrar las escuelas, la enseñanza formal y el trabajo se trasladaron a los hogares, provocando diferentes consecuencias:

\subsection{3.a. Dislocación del tiempo y espacio escolar}

En este contexto muchos adultos enfrentan dificultades para armonizar tiempos y demandas laborales, familiares y escolares, lo que conlleva a altos niveles de frustración, de estrés y un franco desgaste físico y emocional. En el foro se vislumbran narrativas como estas: 
En esta crisis me fui acomodando sobre la marcha, con gusto a abandono, dejando físicamente la institución, los estudiantes y los animales en la chacra escuela, para trabajar desde mi casa, intentando acoplarme a los ritmos de mi familia. (Comunicación personal)

Al mismo tiempo, algunos relatos dan cuenta de la búsqueda de ciertos rituales, la necesidad de establecer un tiempo (una duración, un horario) más o menos separado del resto del tiempo cotidiano, y las consecuentes dificultades para hacerlo: «No puedo separar mi trabajo de mi vida personal. Soy en el mismo espacio y simultáneamente estudiante, docente, mamá y esposa» (Comunicación personal).

\subsection{3.b. Intensificación del trabajo}

La mayoría de los relatos refieren a la prolongación indefinida del tiempo de trabajo para reuniones institucionales, para la preparación de las clases, la selección de los recursos, la evaluación de las producciones de los estudiantes y la atención de los alumnos con mayores dificultades. Reconocen que es un privilegio poder trabajar desde sus hogares pero que esta nueva modalidad representa una «triple jornada laboral». Así, varios señalaron que multiplicaron sus acciones, que la enseñanza en la virtualidad requiere muchísima preparación previa de los materiales, un entorno que permita variabilidad de herramientas y recursos y una tutorización permanente de los aprendizajes.

Además, en general, los docentes se vieron enfrentados a la necesidad de incorporar rápidamente nuevas formas de comunicación con sus alumnos. Desde el primitivo uso del teléfono o de notas entregadas con el apoyo alimentario, hasta mensajes de WhatsApp, videoconferencias y plataformas educativas transformaron sus rutinas y los desbordaron de responsabilidades.

En el foro surgieron relatos, anécdotas, reflexiones y comentarios en los que los docentes manifestaron desarrollar un aluvión de nuevas tareas en su afán por recrearse en la virtualidad: «Me di cuenta de que en casa no tengo recreos ni momentos de ocio. Trato siempre de estar, pero por momentos, la verdad, es una situación agotadora» (Comunicación personal).

\subsection{3.c. Invasión a la intimidad}

Las clases suspendidas impiden que la escuela sea el espacio de trabajo, el afuera, el lugar de encuentro con el otro. Ya no hay afuera. Lo laboral se confunde con lo doméstico. Así lo describe un relato del foro: «Desistí de usar el e-mail porque la mayoría no tenía conexión [...] Mis alumnos tienen mi número de teléfono y ellos o sus padres me contactan a cualquier hora» (Comunicación 
personal). Una docente comenta sobre la incomodidad surgida al recrear el aula en la virtualidad: «Al prender la cámara todos ingresan a mi casa, conocen mi espacio, mis hijos» (Comunicación personal). Las narraciones describen que en las clases fue común escuchar ruidos de la casa o de la calle e interrupciones inesperadas de hijos, familiares y mascotas.

Los relatos del foro dan cuenta que la «domestización de la escuela» (Dussel, 2020b) resultó ser un trabajo complejo y arduo. Describen el agotamiento constante provocado por la intensificación del trabajo y la conexión permanente. Señalan que el «borramiento» de fronteras provocó varios problemas de difícil de solución como complicaciones de vínculos, dificultades para construir redes tanto de aprendizajes como afectivas y obstáculos para suspender la vida cotidiana en el ejercicio profesional.

\subsubsection{Acompañamiento de los procesos de aprendizaje}

En su intento por asegurar la continuidad pedagógica, los docentes dan cuenta de diversas dificultades para recuperar lo propio de la vida escolar en las actuales condiciones tecnológicas. Reconocen que el acto pedagógico no es una simple yuxtaposición de intervenciones individuales, sino una construcción social, es aprender «juntos» con un profesor que acompaña a cada uno en su singularidad. Ese «acompañamiento» de los procesos de aprendizaje en sus alumnos de manera colectiva pero también singular se ve afectado por los siguientes motivos:

\subsection{4.a. Escasa alfabetización digital}

Como señala Area Moreira (2014), educar en el siglo XXI implica el reconocimiento y la incorporación de nuevas alfabetizaciones al sistema educativo. Según este autor, la formación de la ciudadanía en la sociedad informacional debe basarse en la igualdad de oportunidades de acceso a la cultura que se expresa, produce y distribuye a través de múltiples soportes, mediante diversas tecnologías y empleando distintos formatos y lenguajes representacionales. Los docentes participantes, que poseen una formación en el uso de las TIC en contextos educativos, señalan que en sus propuestas pedagógicas integran distintos tipos de lenguajes y modalidades de representación de la información: «En mis clases focalizo en habilidades como la búsqueda y localización de la información a través de diversas tecnologías (sean impresas, audiovisuales o digitales)» (Comunicación personal).

Sin embargo, la mayoría de ellos reconoce que sus alumnos, a pesar de formar parte de una nueva generación vinculada con la tecnología digital, en general no saben aprender con esta; saben usarla para comunicarse, para 
las redes sociales, pero no necesariamente la emplean como un recurso de aprendizaje.

Nuestros alumnos poseen escasas habilidades para abordar las herramientas y aplicaciones digitales como Drive, Classroom, e-mail, chat, etc. Como educadores, somos conscientes de que la producción digital requiere de otros procesamientos y mecanismos, como utilizar buscadores, conocer criterios de búsqueda, de base de datos, saber abrir y guardar archivos, descargar audios, videos, fotos, imágenes, la utilización de procesadores de textos, etc. (Comunicación personal)

Además, las narraciones docentes advierten que cuando las tecnologías se incluyen en las propuestas de enseñanza desde un enfoque crítico, estas se sustentan en supuestos teóricos y metodológicos que respaldan pedagógicamente las decisiones docentes. Sin embargo, aseguran que muchos de sus colegas docentes las han incluido al servicio de formas pedagógicas tradicionales. Como bien expresa una participante del foro, «no podemos pretender que solo es una mudanza de lo presencial a lo virtual» (Comunicación personal). Ellos manifiestan que algunos docentes han intentado aferrarse a sus viejas prácticas y han utilizado las videoconferencias a través de diferentes aplicaciones como única opción para continuar enseñando. Otros, por el contrario, les han pedido asistencia: «La tarea no ha sido sencilla, dado que nos hemos tenido que acompañar y capacitar entre nosotros mismos, sin respuestas ni instancias de formación por parte del Ministerio» (Comunicación personal).

\subsection{4.b. Falta del feedback instantáneo}

Asegurar la continuidad pedagógica de los alumnos implica mantener un contacto permanente con ellos y ofrecerles actividades para consolidar sus logros y estimularlos intelectualmente. Sin embargo, cuando el alumno no está presente y la interacción pedagógica se ve reducida, esto resulta muy difícil, sino imposible. El profesor necesita poder analizar las reacciones a sus propuestas para ayudar a superar los posibles obstáculos. Como han testimoniado muchos, esto es particularmente difícil si no se trabaja en forma presencial: «Nos falta el cara a cara, enviamos las tareas y pasan semanas hasta que aparece una respuesta» (Comunicación personal). Una de las colegas se animó a decir: «No sé si me están siguiendo, si mis propuestas los convocan, porque prácticamente no recibo respuestas» (Comunicación personal). En la virtualidad, el vínculo se vuelve de alguna manera menos controlado, por lo que los docentes expusieron que fue necesario diseñar dispositivos de seguimiento y acompañamiento, establecer otros puentes de contacto y ofrecer diferentes vías de comunicación. 
Además, los profesores señalan que las plataformas de videoconferencia que utilizan, como Zoom y Meet, resultan efectivas para transmitir en una dirección o entre pocos participantes, pero no lo son tanto para seguir hilos de pensamiento más singulares, para generar discusiones e intercambiar miradas.

\subsection{4.c. Dificultad de atención}

En este tiempo, los profesores han descubierto que cuando el proceso de enseñanza y aprendizaje se descontextualiza, se desinstitucionaliza, surgen grandes dificultades pedagógicas. Una de ellas es el problema de la atención en el ámbito doméstico. Los relatos sostienen que existe una dispersión muy fuerte, que es difícil concentrarse en casa. Todo el tiempo lo cotidiano interrumpe los procesos de enseñanza y aprendizaje. Un comentario lo refleja en el foro: «Es difícil administrar mis tiempos de trabajo y focalizar en mi tarea mientras cuido física y emocionalmente de mis niños. Algo similar pasa en casa de mis alumnos» (Comunicación personal).

\subsection{4.d. Dificultad para establecer y sostener vínculos}

Los profesores, a pesar de diseñar actividades movilizadoras, reconocen experimentar dificultades para establecer y sostener vínculos pedagógicos con quienes no estén frecuentemente en contacto con ellos.

Me resulta difícil encontrar maneras de mantener, reforzar y continuar el vínculo pedagógico con mis estudiantes. Todos los días trato de mandarles e-mails y contestar rápido las pocas consultas que recibo para saber cómo están. (Comunicación personal)

Hemos intentado con todos los recursos posibles: WhatsApp, grupos de Facebook, llamadas telefónicas, cualquier cosa para no perderlos. (Comunicación personal)

De los relatos se desprende que para lograr un verdadero acompañamiento en los procesos de aprendizaje de los alumnos, para hacer un andamiaje que sea simultáneamente para todos y para cada uno, hay todavía grandes ventajas en trabajar en un espacio físico donde se ven las caras. A pesar de las dificultades referidas, la mayor coincidencia entre las reflexiones del foro ha sido reconocer que si bien los recursos digitales no reemplazan la potencialidad de los vínculos y métodos de la presencialidad, se constituyen, más allá de este contexto excepcional, en recursos necesarios y enriquecedores de todas las propuestas áulicas. 


\subsection{Cambios implementados}

A pesar de esta migración que estresa a todos, los docentes demuestran haber implementado cambios en sus prácticas, transformaciones en el seguir «haciendo escuela» con la esperanza de que esta se recupere de la tragedia y continúe formando a las nuevas generaciones. Todos reconocen el titánico esfuerzo que están desplegando los educadores, y esto se manifiesta en el foro:

Creo que es muy importante destacar que los docentes en dos semanas pudimos armar un dispositivo de educación no presencial desde cero aprendiendo con amigos, compañeros, hijos y tutoriales, poniendo a disposición de la escuela nuestros propios recursos. (Comunicación personal)

La frustración y la parálisis inicial devinieron en movimientos hacia nuevas formas de trabajo; la incertidumbre y el desconcierto provocado por la desaparición física de las aulas generaron transformaciones en el quehacer escolar. Sin embargo, estos cambios no fueron planeados, no son el resultado de un análisis minucioso y contextualizado de escenarios, de una investigación profunda en la que se contemplaron aspectos positivos y negativos. Fueron producto del instinto, de la inercia.

\subsubsection{Exploración de las posibilidades de las TIC}

Ante el hecho inédito de la pérdida del espacio escolar y del aula, las TIC han abierto grandes posibilidades para continuar los procesos de enseñanza y de aprendizaje. Las clases en tiempos de pandemia se han visto sostenidas por algunos desarrollos de la cultura digital que los docentes han mencionado incluir en sus prácticas:

\subsection{1.a. Incorporación de las TIC para la producción del conocimiento}

En una sociedad atravesada por dispositivos y prácticas digitales diversas, resulta clara la necesidad de repensar la relación de las tecnologías con la producción del conocimiento. Aunque por mucho tiempo fueron resistidas, en esta emergencia que conmovió los cimientos del sistema educativo, las TIC se instalaron definitivamente en la esfera escolar. Los docentes se vieron obligados a incorporar las tecnologías digitales en sus propuestas de enseñanza. Todo este proceso fue un ensayar, probar, discutir, proponer. No hubo certezas. Pero la urgencia de la situación impulsó a los distintos actores de la interfaz educativa a coevolucionar (Scolari, 2018), a formarse en el uso de recursos TIC, a usar internet, videoconferencias, videos y hasta el teléfono para seguir enseñando. Muchos apelaron al uso intensivo de plataformas y redes sociales, otros a reuti- 
lizar los diversos recursos de la web, y algunos se aventuraron a la creación de sus propios contenidos a través de aplicaciones en línea. En poco tiempo, los docentes aprendieron a crear, publicar y compartir objetos de aprendizaje con formatos diversos; comenzaron a resignificar las posibilidades de las TIC para diseñar sus propuestas. No cabe duda de que estamos ante una nueva actitud de los docentes hacia la tecnología, que los acerca a sus alumnos que la habitan con anterioridad. Así los expone un docente:

Necesitábamos que una pandemia que nos trajera al presente y nos empujara a utilizar los medios digitales como herramientas de enseñanza. Los medios digitales están presentes durante todo momento del día de todas las personas; los usamos para todo: para recibir las facturas del agua, de la luz, de internet, de las tarjetas, para hacer pagos, transferencias, para ver el clima, para viajar, etc. Solo faltaba llevarlos al aula. (Comunicación personal)

4.2.1.b. Desarrollo de nuevas formas de sostener vínculos a través de las TIC A diferencia de la clase presencial, donde la copresencia de los cuerpos, las voces y las miradas favorecen el desarrollo de vínculos sobre todo en el nivel primario y secundario, en la no presencialidad fue necesario generar nuevos encuentros periódicos que promuevan un acompañamiento cercano. Los docentes manifestaron confiar en la centralidad de los lazos colectivos, la solidaridad y el cuidado del otro, y haber intentado ser sostén emocional de sus alumnos inventando con el uso de las TIC nuevas cercanías. Al sistematizar los aprendizajes de esta experiencia, resaltaron que han hecho hasta lo imposible por humanizar los encuentros a la distancia, por recuperar el clima y los sentidos del aula. Además, describieron haber implementado diferentes acciones reparadoras, intentando reconectar a los que quedaron fuera del universo institucional educativo e impedir que las desigualdades se profundicen.

En esta realidad inédita los docentes afirman que ha sido sin duda necesario incorporar a sus prácticas el uso de dispositivos alternativos, heterogéneos y polifacéticos como el celular para sostener vínculos: «El celular se convirtió en una herramienta esencial para que los estudiantes puedan "continuar" con el aprendizaje. Algo que para muchos era impensado» (Comunicación personal). Pero la continuidad pedagógica sostenida en este modo significó cambios en el vínculo entre escuela y familia. Si bien se produjo una mayor cercanía y una mayor empatía como consecuencia del conocimiento de la realidad de cada familia, también se estableció una informalidad vincular que disolvió los límites espaciales y temporales del trabajo docente y que tensionó la organización de su actividad. 


\subsection{1.c. Recuperación de aprendizajes invisibles}

Por años, los aprendizajes de habilidades y lógicas no tradicionales a través de recursos no convencionales han sido resistidos debido a la falta de equipamiento de las aulas y la formación tradicional de los docentes. Quizás, como propone Cobo (2016), este sea un buen momento para que la escuela reconozca las formas alternativas de adquirir conocimientos, para recuperar aprendizajes invisibles.

Algunos docentes manifiestan que durante la (AS)DISPO han evidenciado en sus alumnos el inicio de una apropiación tecnológica exploratoria, basada en la curiosidad, en la creatividad y en la construcción de redes informales:

Sugerí a los estudiantes el uso de Padlet para trabajar colaborativamente. Rápidamente comenzaron a explorar la herramienta y a descubrir sus usos y potencialidades. Seguramente podrán hacer lo mismo con otras trabajando con y junto a otros. (Comunicación personal)

Además, varios docentes señalaron que ellos mismos han descubierto diferentes aplicaciones digitales para diseñar sus propios materiales educativos a través de la propia exploración: «Aprendo del prueba y error, porque para poder llegar a todos, voy buscando diferentes modos y medios... busco experiencias de otros colegas y juntos proponemos nuevas estrategias, y así vamos, planeando el día a día» (Comunicación personal).

Los docentes han cambiado su relación con las TIC, han incorporado nuevas formas de enseñar y aprender con ellas. Hoy sostienen con certeza que deben ser parte del contenido de sus propuestas (sin importar la modalidad educativa), que deben usarse para habilitar un diálogo fluido y abierto con los saberes que se producen y circulan en la sociedad. Ha quedado en evidencia que cuando las TIC son adoptadas en un contexto que estimula y reconoce la creación individual y colectiva, con el fin de ampliar las posibilidades de expresión y comunicación, pueden contribuir de manera consistente a enriquecer los procesos de aprendizaje.

\subsubsection{Reconocimiento de la necesidad de desarrollar nuevas prácticas y competencias}

Una de las cuestiones que emergen en este contexto global sin precedentes es la necesidad de desarrollar nuevas competencias. Los docentes revelan que es urgente formar sujetos con capacidades para desenvolverse en entornos cambiantes, dispuestos a aprender de otros y con otros y a emplear la tecnología para generar procesos de aprendizaje colaborativo y no solo como un recur- 
so de aprendizaje individual. Ellos mismos expresan que el papel del docente debe ser el de un organizador y supervisor de las actividades de aprendizaje que los alumnos realizan con las tecnologías: «Debemos planificar el proceso y las actividades de alfabetización como una tarea integrada y transversal en el desarrollo de todas las materias» (Comunicación personal).

\subsection{2.a. Redefinición de prácticas alfabetizadoras}

Una gran coincidencia en las narrativas docentes es que manifiestan haber advertido un cambio en las prácticas escolares de la alfabetización. Declaran que este contexto de adversidad ha generado una redefinición de la alfabetización, ya no solo entendida en lectoescritura, sino también en el desarrollo de competencias audiovisuales, digitales e informacionales.

Esta situación ha puesto en evidencia la necesidad de formar alumnos para que puedan reconstruir y dar significado a la multitud de información que obtienen extraescolarmente a través de tecnologías y desarrollar las competencias para utilizarlas y expresarse. (Comunicación personal)

\subsection{2.b. Implementación de nuevas dinámicas de construcción de saberes} De manera paradójica, el (AS)DISPO multiplicó los encuentros entre colegas, las conversaciones, los espacios de intercambio. A pesar del agotamiento, de la exigencia y del esfuerzo que demanda enseñar en la no presencialidad, las reuniones entre los equipos de trabajo aumentaron. De estos equipos surgió la necesidad de repensar las experiencias escolares a la luz de los nuevos modos de producción y circulación del conocimiento y de socialización. Emergió la necesidad de prácticas docentes colectivas para el desarrollo de trabajos por proyectos interdisciplinarios y por problemas. Una docente del nivel medio expone:

Diseñamos secuencias que involucraban a varios profesores, elaboramos cronogramas para explicitar el tiempo destinado a cada actividad, definimos las actividades que van a hacer los y las estudiantes y aquellas que realizarán los y las docentes. Trabajamos en equipo. (Comunicación personal)

La pandemia ha demostrado la necesidad de plantear una metodología de enseñanza que favorezca procesos de aprendizaje a través de proyectos donde los propios alumnos articulen planes de trabajo y desarrollen acciones con las tecnologías para resolver problemas relevantes y con significado. Es momento, como propone Cobo, de «abrir espacios de deconstrucción y reconstrucción de nuevos conocimientos en diversos formatos y lenguajes, 
atribuyendo un mayor protagonismo al sujeto que aprende, estimulando el pensamiento crítico y la experimentación» (Cobo, 2016: 35).

\subsection{2.c. Reestructuración de contenidos}

En este escenario surgieron interrogantes como ¿es posible continuar enseñando el programa establecido en marzo?, ¿qué contenidos y qué tipo de actividades habría que privilegiar? La preocupación por la continuidad pedagógica se incorporó rápidamente en los debates académicos y la emergencia disparó la necesidad de responder con rapidez a situaciones imprevistas. Se generaron protocolos, instructivos, manuales y recomendaciones para organizar la vida institucional. Con todas las dificultades e improvisaciones, las pedagogías de la emergencia revelaron la necesidad de reflexionar sobre las prácticas de enseñanza, sobre los contenidos seleccionados, las actividades propuestas y su desafío intelectual, sobre la organización de los grupos y de los tiempos de trabajo. Los docentes aseguran que fue necesario recordar que los saberes valiosos no se reducen al programa escolar. Así, los educadores manifiestan que debieron establecer principios que sustentaran sus propuestas como el de no estandarizar, sino responder según los contextos, ajustar sus planificaciones a las realidades particulares y dosificar tareas. Una docente de nivel medio expresa:

Tuvimos que realizar readecuaciones curriculares importantes, concentrar las actividades en los saberes prioritarios. De poco sirve intentar trabajar con todos los contenidos planificados con la secreta esperanza de que finalmente el alumno los alcance. (Comunicación personal)

Los profesores del nivel superior, por su parte, también afirmaron que en ocasiones optaron por una readecuación de los contenidos de sus materias o bien por un recorte y/o por una reasignación de los mismos apuntando a su desarrollo en otras materias vinculadas.

La velocidad con que hubo que resolver esta situación inesperada no hizo posible todavía una evaluación de los cambios puestos en marcha. Sin embargo, podemos afirmar que muchos de los cambios en la relación entre educación y tecnologías experimentados pueden enriquecer la vuelta a la presencialidad.

Sabemos que las tecnologías atraviesan y modifican a las sociedades. Como sostiene Litwin (2000), aprender con tecnologías ofrece oportunidades cognitivas diferentes a las que se generan al aprender sin ellas. Las dificultades enfrentadas y las improvisaciones implementadas lo han puesto en evidencia. La integración crítica de las TIC a la educación permite diversificar, problematizar y potenciar las posibilidades de aprender y de enseñar. 


\section{Conclusiones}

Francesco Tonucci decía, en una entrevista en El País, que habría que hacer de esta experiencia una oportunidad de aprender: «Aprovechemos para pensar si otra escuela es posible» (Tonucci, 2020). Por supuesto, todo el mundo está de acuerdo en que habrá un antes y un después de la pandemia, pero nadie sabe qué características tendrá este «después». Sin embargo, la percepción de educandos y educadores es que esta es una gran posibilidad de aprendizaje. Un enunciado así lo expresa en el foro:

Sin duda, cuando todo esto termine no seré la misma docente, ya que esta situación me ha invitado a pensar una y otra vez en otras propuestas de enseñanza, en otras formas de comunicación, en otra escuela posible. (Comunicación personal)

Afortunadamente, el COVID-19 no detuvo a la escuela; solo la trasladó, la llevó a la casa. No podemos afirmar que colaboró en la implementación de una educación en línea, virtual o a distancia ya que faltó planificación y una verdadera articulación de equipos de trabajo. Pero la situación de pandemia no le quitó las esperanzas de un mejor futuro. Las descripciones en el foro coinciden en que esta ha sido una gran oportunidad para promover la solidaridad, la acción colectiva, la empatía por el otro, y para establecer un nuevo sistema educativo inspirado en la cooperación y en el trabajo comunitario. Todos los docentes reconocen que durante la (AS)DISPO incrementaron sus contactos con colegas y equipos directivos para consensuar el curso de acciones a seguir y trabajar en proyectos mancomunados.

Estos meses transcurridos en un escenario tan excepcional han cambiado el ecosistema escolar. Alguien expresa con alegría: "Ya no somos los mismos. No miramos al mundo con los mismos ojos. Este original momento que vivimos es desconcertante pero a la vez muy emocionante por todo lo que está generando» (Comunicación personal). El colectivo de docentes, con la heterogeneidad que lo caracteriza, ha desarrollado en poco tiempo distintas transformaciones en sus prácticas; no solo transformaciones de espacio y de tiempo, sino también una reorganización de saberes, una redefinición de la comunicación y una valiosa reinterpretación de los modos en los que las tecnologías pueden (y deben) ser incluidas en diferentes propuestas orientadas por el sentido pedagógico.

Resulta fundamental comenzar por sistematizar los aprendizajes que esta experiencia deja. Entre muchos otros, el reconocimiento de la prioridad absoluta que debe asumir el Estado de realizar una importante inversión en 
políticas públicas orientadas al fortalecimiento del sistema educativo, en busca de garantizar mayores niveles de igualdad. La escuela es irremplazable y demanda ser reinventada. Pero para hacerlo, como plantea Paulo Freire (2019), hay que poder mirar la realidad críticamente y poder entender los problemas que tenemos como sociedad, como comunidad, y educar sujetos que tengan la posibilidad de transformar eso desde una perspectiva crítica.

Otro gran aprendizaje es que ya no es posible seguir pensando en un único modelo de escuela. Es necesario pensar en nuevas formas de escolaridad, híbridas, donde lo virtual y lo presencial coexistan, donde se incluya a las familias, donde las instituciones funcionen tanto en espacios reales como virtuales y procuren la formación de sujetos con capacidades para desenvolverse en entornos cambiantes, ciudadanos digitales dispuestos a aprender de otros y con otros a través de la adopción tecnológica. Y para ello el rol del Estado también es fundamental ya que deberá desarrollar políticas públicas que contemplen nuevos regímenes laborales docentes, capacitaciones adecuadas y dotación de recursos TIC a las instituciones.

La complejidad del proceso en curso demanda habilitar espacios de discusión desde donde surjan propuestas con sentido democrático e igualitario que propongan pensar este tiempo y construir otros futuros, caminos a seguir para aprovechar mejor el potencial educativo de las tecnologías digitales. Hoy más que nunca necesitamos documentar las experiencias vividas en esta pandemia, compartirlas, analizarlas y pensar conjuntamente en la reconstrucción de la escuela. «Y tal vez para eso están las escuelas, para que un buen día algo se reconstruya, para que un buen día nunca sea demasiado tarde» (Skliar, 2020). 


\section{Referencias bibliográficas}

AREA MOREIRA, M. (2014). La alfabetización digital y la formación de la ciudadanía del siglo XXI. Revista Integra Educativa, 7(3), 21-33.

COBO, C. (2016). La innovación pendiente: Reflexiones (y provocaciones) sobre educación, tecnología y conocimiento. Montevideo: Colección Fundación Ceibal/ Debate.

DUSSEL, I. (2020a). La escuela en la pandemia. Reflexiones sobre lo escolar en tiempos dislocados. Práxis Educativa, 15, 1-16.

DUSSEL, I. (2020b). La clase en pantuflas. Reflexiones a partir de la excepcionalidad. En Maggio, M. y otros. Bitácora de cuarentena para docentes y pedagogues. S/d.

DUSSEL, I.; Ferrante, P. y Pulfer, D. (2020). Pensar la educación en tiempos de pandemia. Entre la emergencia, el compromiso y la espera. Buenos Aires: UNIPE Editorial Universitaria.

FREIRE, P. (2019). Pedagogía de los sueños posibles: Por qué docentes y alumnos necesitan reinventarse en cada momento de la historia. Buenos Aires: Siglo XXI Editores.
LITWIN, E. (comp.). (2000). Educación a distancia. Temas para el debate en una nueva agenda educativa. Buenos Aires: Amorrortu. SCOLARI, C.A. (2018). Las leyes de la interfaz: Diseño, ecología evolución, tecnología (vol. 141). Barcelona: Editorial Gedisa.

SCOLARI, C. (2019). ¿Cómo analizar una interfaz? Method January 2019. Disponible en: https://www.researchgate.net/publication/330651740 Como analizar una interfaz

SKLIAR, C. (2020). Acerca de extrañar la escuela. Charla con Kaplan, C.; Feldfeber, M. y Catelli, J., Facultad de Filosofía y Letras, UBA. S/d.

TERIGI, F. (2020). Enseñar y aprender en tiempos de pandemia. En Maggio, M. et al. Bitácora de cuarentena para docentes y pedagogues. S/d.

TONUCCI, F. (2020). No perdamos este tiempo precioso dando deberes. Diario El País, 11 de abril de 2020. Disponible en: https:// elpais.com/sociedad/2020-04-11/francesco-tonucci-no-perdamos-este-tiempo-precioso-dando-deberes.html [acesso: 21/08/2020]. 